\title{
Structural Genomics
}

National Cancer Institute

\section{Source}

National Cancer Institute. Structural Genomics. NCI Thesaurus. Code C20126.

The combination of computational DNA sequence analysis with structural determinations by X-ray crystallography and NMR techniques to study protein structural families. (from RFA: GM-99-009) 